\title{
Spectinomycin Hydrochloride Anhydrous
}

National Cancer Institute

\section{Source}

National Cancer Institute. Spectinomycin Hydrochloride Anhydrous. NCI Thesaurus. Code C90690.

The anhydrous hydrochloride salt form of spectinomycin, an aminocyclitol aminoglycoside antibiotic derived from Streptomyces spectabilis with bacteriostatic activity. Spectinomycin binds to the bacterial 30 S ribosomal subunit. As a result, this agent interferes with the initiation of protein synthesis and with proper protein elongation. This eventually leads to bacterial cell death. 